\title{
OPEN Identification of differentially methylated genes in first-trimester placentas with trisomy 16
}

Ekaterina N. Tolmacheva ${ }^{1 凶}$, Stanislav A. Vasilyev ${ }^{1}$, Tatiana V. Nikitina ${ }^{1}$, Ekaterina S. Lytkina ${ }^{2}$, Elena A. Sazhenova ${ }^{1}$, Daria I. Zhigalina ${ }^{1}$, Oksana Yu. Vasilyeva ${ }^{1}$, Anton V. Markov ${ }^{1}$, Victoria V. Demeneva ${ }^{1}$, Liubov A. Tashireva ${ }^{3}$, Anna A. Kashevarova ${ }^{1}$ \& Igor N. Lebedev ${ }^{1}$

The presence of an extra chromosome in the embryo karyotype often dramatically affects the fate of pregnancy. Trisomy 16 is the most common aneuploidy in first-trimester miscarriages. The present study identified changes in DNA methylation in chorionic villi of miscarriages with trisomy 16 . Ninetyseven differentially methylated sites in 91 genes were identified (false discovery rate (FDR) $<0.05$ and $\Delta \beta>0.15$ ) using DNA methylation arrays. Most of the differentially methylated genes encoded secreted proteins, signaling peptides, and receptors with disulfide bonds. Subsequent analysis using targeted bisulfite massive parallel sequencing showed hypermethylation of the promoters of specific genes in miscarriages with trisomy 16 but not miscarriages with other aneuploidies. Some of the genes were responsible for the development of the placenta and embryo (GATA3-AS1, TRPV6, SCL13A4, and $C A L C B$ ) and the formation of the mitotic spindle (ANKRD53). Hypermethylation of GATA3-AS1 was associated with reduced expression of GATA3 protein in chorionic villi of miscarriages with trisomy 16. Aberrant hypermethylation of genes may lead to a decrease in expression, impaired trophoblast differentiation and invasion, mitotic disorders, chromosomal mosaicism and karyotype self-correction via trisomy rescue mechanisms.

The first trimester of pregnancy is the most critical period for embryo survival. At 8-10 weeks of pregnancy, cytotrophoblast cells invade the decidualized endometrium, results in remodeling of the spiral arteries of the uterus. Disruption of normal trophoblast differentiation and development and gestational remodeling of the uterine spiral arteries leads to a wide range of obstetric complications, from delayed fetal growth to fetal death and/or preeclampsia.

Mosaic trisomy may cause serious defects in physical and cognitive development ${ }^{1}$, which indicates that chromosomal aneuploidy has a harmful pleiotropic effect. The increased genes on the additional chromosome itself causes a genome-wide imbalance in gene activity. Aneuploidies of different chromosomes change the epigenetic profile of various tissues, including the placenta ${ }^{2}$.

Trisomy of chromosome 16 is the most common aneuploidy in miscarriages of the first trimester of pregnancy, and it accounts for approximately $6 \%$ of all miscarriages ${ }^{3}$. Most miscarriages with trisomy 16 are empty fetal sacs or disorganized embryos with minimal embryonic development ${ }^{4}$. Miscarriages with trisomy 16 also have abnormal chorionic villous morphology with a cystic, clubbed, or hypoplastic appearance. Mosaic forms of this aneuploidy occur later in pregnancy and postnatally in rare cases ${ }^{5}$. Mosaic forms of trisomy 16 are associated with a high probability of fetal death, preterm birth, intrauterine growth retardation, fetal abnormalities, and preeclampsia ${ }^{6}$. These malformations likely occur due to the aberrant expression of imprinted genes located on chromosome $16^{7,8}$.

Aneuploidies are also often characterized by a disturbance of the genome methylation profile, which leads to developmental disorders and embryonic death ${ }^{2}$. Epigenetic modifications in the placentas of fetuses with mosaic trisomy 16 and their relationship with early-onset preeclampsia were studied in detail previously ${ }^{9}$. However, data on DNA methylation in trisomy 16 in the first trimester of pregnancy, during which there is active placental differentiation and invasion of endovascular cytotrophoblasts into the spiral arteries, are scarce.

Trisomy 16 in first-trimester miscarriages primarily originates from segregation errors in maternal meiosis $\mathrm{I}^{10}$. Therefore, the formation of the body's epigenetic program after conception occurs against a background of

\footnotetext{
${ }^{1}$ Research Institute of Medical Genetics, Tomsk National Research Medical Center, Tomsk, Russia. ${ }^{2}$ National Research Tomsk State University, Tomsk, Russia. ${ }^{3}$ Cancer Research Institute, Tomsk National Research Medical Center, Tomsk, Russia. ${ }^{\varpi}$ email: kate.tolmacheva@medgenetics.ru
} 
a

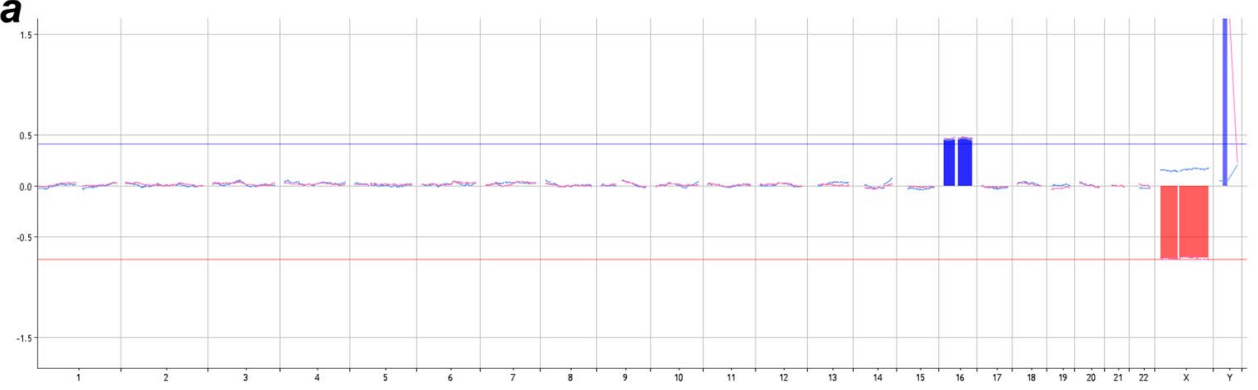

b

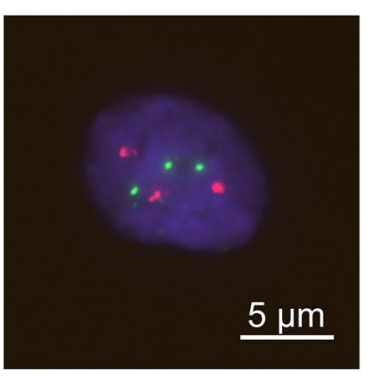

Figure 1. Results of cytogenetic analysis of chorionic villous trophoblasts of miscarriages with trisomy 16. (a) Example of the aCGH profile in chorionic villous trophoblasts with trisomy 16. (b) Example of chorionic villous trophoblasts with trisomy 16 based on the results of FISH analysis with two-color subtelomeric DNA probes specific to $16 \mathrm{p}$ (red) and 16q (green). Interphase nuclei were stained with DAPI (blue).

trisomy 16 in the zygote and later in most cells, even in chromosomal mosaicism. Aneuploidy in this case is a causative factor for the induction of epigenetic changes in the genome. The present study identified differentially methylated genes in chorionic villi of first-trimester miscarriages with trisomy 16.

\section{Results}

The study was performed in two stages. In the first stage, we performed a large-scale analysis of the DNA methylation profile in chorionic villi in 14 miscarriages with trisomy 16 and 6 induced abortions with a normal karyotype using the Infinium HumanMethylation27 BeadChip (Illumina). This stage identified numerous genes with differentially methylated CpG sites in miscarriages with trisomy 16 . Using targeted bisulfite massive parallel sequencing, we analyzed the level of DNA methylation of the promoter regions of 5 differentially methylated genes on the expanded samples of miscarriages with trisomy $16(n=29)$. Only miscarriages with a normal karyotype and trisomy on other chromosomes were included in the study at this point because we were interested in the specificity of identified differential methylation of these genes for trisomy 16.

Samples of extraembryonic tissues of miscarriages and induced abortions were obtained from the biocollection of the Research Institute of Medical Genetics, Tomsk NRMC. These tissues were collected for 15 years and stored at a temperature of $-80^{\circ} \mathrm{C}$. The criteria for inclusion in the study were the karyotype of miscarriage and gestational age from 6 to 12 weeks. The age of the parents was not specifically controlled.

Genome-wide DNA methylation in chorionic villous trophoblasts with trisomy 16. Trisomy 16 was discovered in chorionic villous trophoblasts of miscarriages using conventional karyotyping and array comparative genomic hybridization $(\mathrm{aCGH})$ and validated further using interphase fluorescent in situ hybridization (FISH) (Fig. 1). The DNA methylation indices were analyzed in 27,578 CpG sites of 14,523 genes in chorionic villi of 14 miscarriages with trisomy 16 and 6 induced abortions with normal karyotypes. With the cutoff false discovery rate (FDR) $<0.05,172$ hypomethylated and 777 hypermethylated CpG sites were identified in miscarriages with trisomy 16 . CpG sites with a $\Delta \beta>0.15$ were considered biologically significant differentially methylated sites (DMSs). With this cutoff, 2 hypomethylated sites in 1 gene and 94 hypermethylated CpG sites in 90 genes were identified. Hypermethylation of multiple CpG sites was detected in 4 genes (Supplementary Table S1). Twenty-three of these sites were located inside the CpG islands and 71 were not, but most of the differentially methylated sites were located in the regulatory regions of the genes. No chromosome-specific DMS enrichment was found, and only 4 differentially methylated genes (DMGs) were located on chromosome 16, which corresponded to the average level on other chromosomes (Supplementary Fig. S1).

We performed enrichment analysis of the $90 \mathrm{DMGs}$ and found significant enrichment of a cluster of 47 genes encoding secreted proteins $(\mathrm{p}=2.80 \mathrm{E}-08)$, signaling peptides $(\mathrm{p}=6.50 \mathrm{E}-08)$, and receptors with disulfide bonds $(\mathrm{p}=2.20 \mathrm{E}-06)$ (DAVID, UniProt, enrichment score: 8.76). Twenty-four of these genes were included in all 3 functional groups (Supplementary Fig. S1).

Among the DMGs in miscarriages with trisomy 16, two genes (FOSL1 and PCDH12) were involved in placental development (GO:0001890). When the DMGs in miscarriages with trisomy 16 and genes involved in placental development (GO:0001890) were analyzed using STRING, 25 DMGs had functional connections with genes involved in placental development (score >0.90, Fig. 2).

For a more rigorous identification of biologically significant DMGs, we used a criterion of $\Delta \beta>0.2$ and revealed hypermethylation in the promoters of 17 genes and hypomethylation of 1 gene (Table 1).

Detailed analyses of the DNA methylation of the identified DMGs. We analyzed the methylation indices of the promoter regions of 5 DMGs (ANKRD53, TRPV6, GATA3-AS1, SCL13A4, and CALCB) in chorionic villi trophoblasts of miscarriages with trisomy 16 and induced abortions with a normal karyotype using targeted bisulfite massive parallel sequencing (Table 2). Differentially methylated sites were located in the CpG islands for 3 genes: ANKRD53, GATA3-AS1, and CALCB.

Hypermethylation of various fractions of the analyzed $\mathrm{CpG}$ sites in the promoters of all 5 genes in miscarriages with trisomy 16 was found $(\mathrm{p}<0.05)$ (Table 2$)$. No hypomethylated CpG sites were observed. Therefore, 


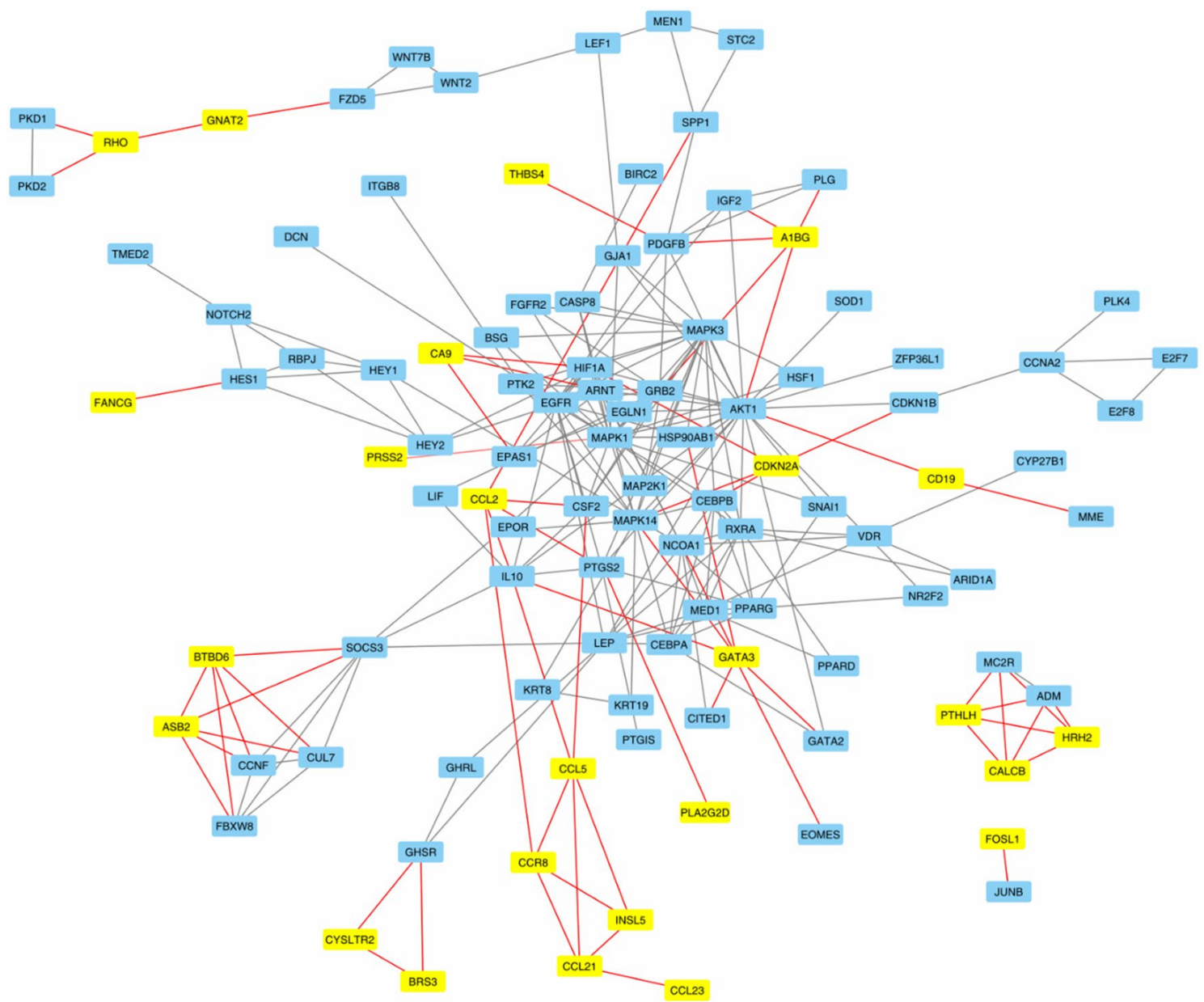

Figure 2. Functional relationships between the products of genes hypermethylated in chorionic trophoblasts of embryos with trisomy 16 (highlighted in yellow) and the products of genes involved in the development of the placenta (placental development, GO: 0001890) (highlighted in blue) (STRING database, score >0.90). The connections of the products of the hypermethylated genes with the proteins involved in the development of the placenta are highlighted in red.

the results obtained from targeted bisulfite massive parallel sequencing confirmed the results of the genome-wide DNA methylation analysis, which was performed using the Infinium HumanMethylation27 BeadChip.

The increased methylation indices of the studied genes may also be associated with aneuploidy on other chromosomes. To exclude this effect, we compared the methylation indices of the ANKRD53 and GATA3-AS1 genes in 26 miscarriages with aneuploidy other than trisomy 16 (trisomy 2, 7, 9, 11, 13, 14, 15, 18, 20, 21, or 22 or monosomy of chromosome 13 or the $\mathrm{X}$ chromosome) and 8 induced abortions with a normal karyotype. A similar comparison was made for the TRPV6, SCL13A4, and CALCB genes with miscarriages with trisomy $2(\mathrm{n}=1)$ and trisomy $22(\mathrm{n}=2)$. Only 1 hypermethylated site was identified for GATA3-AS1, and no DMS was found for ANKRD53 (Fig. 3).

Dependence of the gene methylation indices on gestational age and chromosome mosaicism. We analyzed the correlations between the gene methylation indices of 5 DMGs: ANKRD53, TRPV6, GATA3-AS1, SCL13A4, and CALCB. The methylation indices of the GATA3-AS1, SCL13A4, and TRPV6 genes significantly correlated with each other (Fig. 4).

The methylation profile of the normal placenta changes with increasing gestational age ${ }^{12}$. Therefore, we analyzed the dependence of the CpG site methylation indices of all 5 DMGs in miscarriages with trisomy 16 on gestational age. A positive correlation was observed between gestational age and the level of GATA3-AS1_4-5 region methylation (Fig. 4).

The level of mosaicism of trisomy 16 was analyzed in some of the miscarriages $(n=30)$. Eighteen embryos had a pure form of trisomy 16 , and the other embryos $(n=12)$ had mosaicism of trisomy 16 , with a percentage of aneuploid clones ranging from 10 to $90 \%$. The most hypermethylated gene in chorionic villi of miscarriages with trisomy $16(\Delta \beta=0.27$, Table 1$), A N K R D 53$, encodes a protein that is involved in the mechanism of chromosome segregation in mitosis and may cause mitotic nondisjunction, which leads to chromosome mosaicism. However, the methylation indices of the ANKRD53 gene promoter, and the promoters of the other four genes, did not differ between the groups with mosaic and pure forms of trisomy 16 (Fig. 4). This finding may be explained by the fact 


\begin{tabular}{|c|c|c|c|c|c|}
\hline Symbol & RefSeq & Product & CpG-island & $\Delta \beta$ & Chr \\
\hline ZNF804B & NM_181646.2 & Zinc-finger protein $804 \mathrm{~B}$ & True & 0.279 & 7 \\
\hline ANKRD53 & NM_024933.2 & Ankyrin repeat domain-containing protein 53 & True & -0.275 & 2 \\
\hline TRPV6 & NM_018646.2 & Transient receptor potential cation channel; subfamily V; member 6 & False & -0.268 & 7 \\
\hline SEC31L2 & NM_015490.3 & S. cerevisiae SEC31-like 2 isoform a & True & -0.258 & 10 \\
\hline CCL2 & NM_002982.3 & Small inducible cytokine A2 precursor & False & -0.233 & 17 \\
\hline GATA3-AS1 & NM_207423.1 & GATA3 antisense RNA 1 & True & -0.226 & 10 \\
\hline SLC13A4 & NM_012450.2 & Solute carrier family 13 (sodium/sulfate symporters); member 4 & False & -0.223 & 7 \\
\hline SLC17A4 & NM_005495.1 & Solute carrier family 17 (sodium phosphate); member 4 & False & -0.221 & 6 \\
\hline CYSLTR2 & NM_020377.2 & Cysteinyl leukotriene receptor 2 & False & -0.219 & 13 \\
\hline ZNF683 & NM_173574.1 & Zinc-finger protein 683 & False & -0.216 & 1 \\
\hline$C A L C B$ & NM_000728.3 & Calcitonin-related polypeptide; beta & True & -0.215 & 11 \\
\hline PDZD3 & NM_024791.2 & Sodium-phosphate cotransporter IIa C-terminal-associated protein 2 & False & -0.214 & 11 \\
\hline CCR8 & NM_005201.2 & Chemokine (C-C motif) receptor 8 & False & -0.210 & 3 \\
\hline FANCG & NM_004629.1 & Fanconi anemia; complementation group G & True & -0.209 & 9 \\
\hline KRTAP10-8 & NM_198695.1 & Keratin-associated protein $10-8$ & False & -0.204 & 21 \\
\hline$B R S 3$ & NM_001727.1 & Bombesin-like receptor 3 & False & -0.203 & $\mathrm{X}$ \\
\hline CCL21 & NM_002989.2 & Small inducible cytokine A21 precursor & False & -0.202 & 9 \\
\hline SLC $3 A 1$ & NM_000341.2 & Solute carrier family 3 ; member 1 & False & -0.201 & 2 \\
\hline
\end{tabular}

Table 1. Differentially methylated genes (FDR cutoff of $\mathrm{p}<0.05$ and $\Delta \beta>0.2$ ) in chorionic villi of miscarriages with trisomy 16.

\begin{tabular}{|l|l|l|l|l|l|}
\hline Region & Strand & Genomic coordinates (hg19) & CpG & DMS & DMS/all CpG sites, \% \\
\hline ANKRD53_1 & + & chr2: 71206056-71206605 & 40 & 6 & 15 \\
\hline & & ANKRD53 in total & 40 & 6 & 15 \\
\hline CALCB_1 & + & chr11: $15094027-15094302$ & 15 & 3 & 20 \\
\hline CALCB_3 & + & chr11: 15094516-15094823 & 8 & 4 & 50 \\
\hline CALCB_4 & + & chr11: $15094798-15095066$ & 12 & 4 & 33 \\
\hline & & CALCB in total & 35 & 11 & 31 \\
\hline GATA3_1 & + & chr10: $8091317-8091687$ & 30 & 0 & 0 \\
\hline GATA3_2 & + & chr10: $8092469-8093016$ & 57 & 0 & 0 \\
\hline GATA3_4 & + & chr10: $8093670-8094118$ & 37 & 21 & 57 \\
\hline GATA3_5 & + & chr10: $8094186-8094771$ & 32 & 23 & 72 \\
\hline GATA3_8 & + & chr10: $8095096-8095448$ & 15 & 3 & 20 \\
\hline & & GATA3-AS1 in total & 84 & 47 & 56 \\
\hline SLC13A4_1 & - & chr7: 135412984-135413410 & 11 & 0 & 0 \\
\hline SLC13A4_2 & - & chr7: 135412563-135413021 & 7 & 6 & 86 \\
\hline & & SCL13A4 in total & 18 & 6 & 33 \\
\hline TRPV6_1 & - & chr7: 142583430-142583865 & 5 & 0 & 0 \\
\hline TRPV6_2 & - & chr7: $142582950-142583429$ & 11 & 6 & 54 \\
\hline TRPV6_3 & - & chr7: 142582830-142583240 & 6 & 5 & 83 \\
\hline & TRPV6 in total & 22 & 11 & 50 \\
\hline
\end{tabular}

Table 2. Localization and methylation of CpG sites of 5 differentially methylated genes analyzed by targeted bisulfite massive parallel sequencing in chorionic villi of miscarriages with trisomy 16.

that trisomy 16 miscarriages have a predominantly meiosis I origin. Therefore, aneuploid-diploid mosaicism in embryos with trisomy 16 results from a postzygotic trisomy rescue mechanism. These results suggest that human embryos with trisomy 16 activate epigenetic programs to induce their own karyotype self-correction.

One of the analyzed hypermethylated genes, GATA3-AS1, enhances the expression of the key transcription factor GATA3, which controls trophoblast differentiation ${ }^{13}$. Therefore, we compared the expression of the GATA3 protein in chorionic villi of miscarriages with trisomy 16, trisomy 22, and normal karyotypes. The level of GATA3 protein expression was reduced in miscarriages with trisomy 16 compared to miscarriages with trisomy 22, and miscarriages with a normal karyotype had an intermediate level of GATA3 expression (Fig. 5). 


\section{Discussion}

Only one previous study examined the methylation profile in fetuses with trisomy 16 in the first and third trimesters of pregnancy, in which disturbances of placental DNA methylation in the third trimester and the association of aberrant methylation of certain genes with early-onset preeclampsia were studied in detail $^{9}$. However, changes in genome methylation in the placentas of miscarriages in the first trimester were not fully analyzed.

We reviewed our earlier data on the analysis of genome-wide methylation of chorionic villi of first-trimester miscarriages with trisomy 16 and induced abortions using the Infinium HumanMethylation27 BeadChip (Illumina $)^{14}$. Our results confirmed earlier findings that trisomy 16 was associated with an increase in the methylation index of several genes in the chorionic villi, and this increased methylation may play a role in embryo death in the first trimester of pregnancy.

The presence of significant correlations between increased methylation of individual gene promoters and genome hypermethylation, and the fact that the LINE-1 retrotransposon methylation index is increased in the chorionic villi of miscarriages with trisomy 16 compared to induced abortions ${ }^{15}$, indicate that the hypermethylation of DNA in trisomy 16 may be specific to the whole genome. This process is likely triggered by specific gene(s) that are located on chromosome 16. These genes may be involved in the regulation of DNA methylation. According to the Gene Ontology database, several genes involved in DNA and histone methylation are located on chromosome 16. These include four histone methyltransferases (SETD6, SETD1A, PRDM7, and PRMT7), methyltransferase coactivators (CREBBP and PAGR1), and the key chromatin insulator CTCF. Histone and DNA methylation are often linked in repetitive centromeric and subcentromeric sequences ${ }^{16}$, but it is difficult to explain how an increase in the number of genes of histone methyltransferases and their cofactors on chromosome 16 induce an increase in the level of DNA methylation in gene promoters.

The present study found an increase in the DMG methylation index in miscarriages with pure and mosaic trisomy, regardless of the proportion of aneuploid clone cells, which indicates the simultaneous generation of an increased methylation index of the studied genes in the zygote or at the cleavage stage due to a disturbance of the normal mechanisms of hypomethylation ${ }^{17}$. The correlation of the methylation index of some gene regions with gestational age suggests that the increase in the methylation index of the studied genes is not a one-time event but develops gradually and increases with the age of the embryo. The level of DNA methylation may increase on a background of trisomy in gametes or during early development then further increases during the course of pregnancy. This assumption is supported by the increase in the level of genome methylation over the course of normal pregnancy ${ }^{12}$. An earlier study also found increased levels of gene promoter methylation in the placentas of fetuses with trisomy 16, and this increase was more significant than fetuses with a normal karyotype. Eighty-five hypermethylated sites were identified in the placentas of first-trimester trisomy 16 embryos, and approximately $1,195 \mathrm{CpG}$ sites were identified in the third-trimester placentas compared to placentas with a normal karyotype 9 .

Among the hypermethylated genes in chorionic villi of miscarriages with trisomy 16, the GATA3-AS1 gene is particularly interesting. This lncRNA gene is adjacent to GATA3 and is a positive regulator of GATA3 transcription $^{13}$. GATA3 is a transcription factor that is selectively expressed in the trophectoderm at the blastocyst stage of embryo development. GATA3 knockdown leads to a significant decrease in migration and a decrease in HTR8/SVneo cell invasion ${ }^{18}$. GATA3 is essential for the development of trophectoderm clones and maintaining the self-renewal of trophoblastic stem cells ${ }^{19}$. Together with GATA2, GATA3 is involved in the regulation of the a subunit of chorionic gonadotropin, syncytin, HLA-G, and several other genes in human trophoblast cells ${ }^{19}$. DNA methylation regulates the GATA3 gene during human embryonic stem cell differentiation ${ }^{20}$. However, its methylation status during trophoblast differentiation is not clear. Due to the limited available material of miscarriages in our study, only the DNA methylation profile was studied without analysis of the transcription profile of genes. Therefore, we confirmed the association of GATA3-AS1 hypermethylation and reduced GATA3 protein levels in chorionic villi sections in miscarriages with trisomy 16 compared to controls.

The genes of various secreted factors, their receptors, and signal peptides were predominantly hypermethylated in the chorionic villi of miscarriages with trisomy 16 in our study. These genes included TRPV6, SLC13A4, and CALCB. The TRPV6 gene encodes a member of a family of multipass membrane proteins that function as calcium channels. TRPV6 is expressed in the human placenta, primarily in trophoblasts ${ }^{21}$, and its expression in the placenta and fetal bone tissue persists throughout mouse pregnancy ${ }^{22}$. The CALCB gene encodes calcitonin-related beta-peptide (CGRP), which is a neurotransmitter. Its receptors were identified on human trophoblast cells. CGRP is involved in the morphological and functional differentiation of trophoblast cells via CGRP-induced accumulation of intracellular cAMP ${ }^{23}$. SLC13A4 is one of the most common sulfate transporters, and it is expressed primarily in the placenta in syncytiotrophoblast and cytotrophoblast cells ${ }^{24}$. Knockout of this gene in mouse embryos leads to severe fetal abnormalities and prenatal death ${ }^{25}$. Hypermethylation of these genes may lead to inhibition of their expression and disturbance of the normal development and differentiation of the trophoblast, death of embryos with pure trisomy 16 in the early stages of development, or malformations of fetuses with mosaic forms of trisomy 16 that survive until the third trimester.

Mosaic trisomy 16 is a consequence of trisomy rescue, a phenomenon with unclear causes. Aneuploidy itself causes chromosomal instability because of defects in cell cycle progression ${ }^{26}$ or cell proliferation ${ }^{27}$. The level of anaphase lagging is significantly higher in cells with trisomy 7 or 13 than diploid cells due to errors in cytokinesis. This phenomenon is associated with an increase in the concentration of the spartin protein, which is localized in centrosomes and microtubules during mitosis. The SPG20 gene encoding this protein is located on chromosome $13^{28}$.

For trisomy 16, we found hypermethylation of two genes that are involved in mitosis, ANKRD53 and CDKN1A (Supplementary Table S1). ANKRD53 protein localizes to the microtubules of the mitotic spindle around the centrosome in prophase and prometaphase and at the spindle poles in metaphase and anaphase, and it regulates the polymerization of microtubules and cytokinesis ${ }^{29}$. Defects in the methylation of the ANKRD53 gene may 

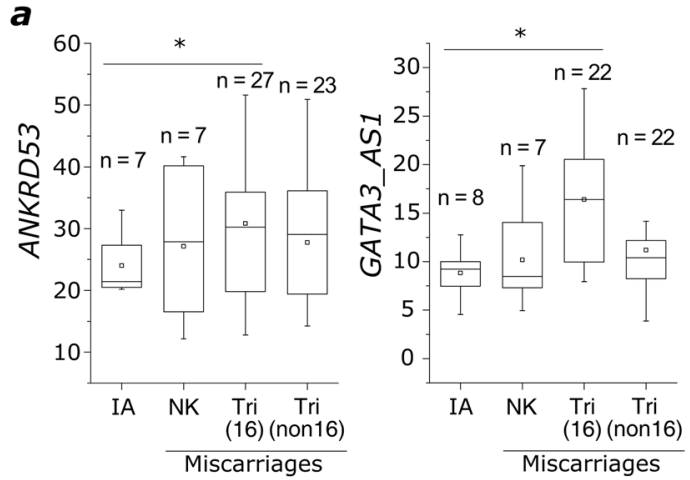

$\boldsymbol{b}$

ANKRD53

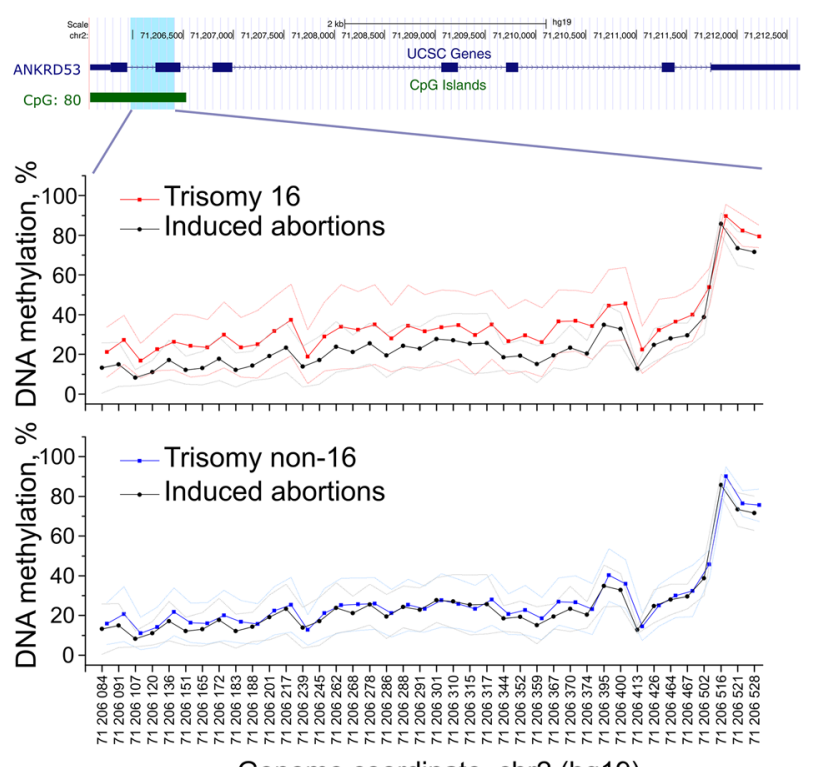

Genome coordinate, chr2 (hg19)

\section{SLC13A4}

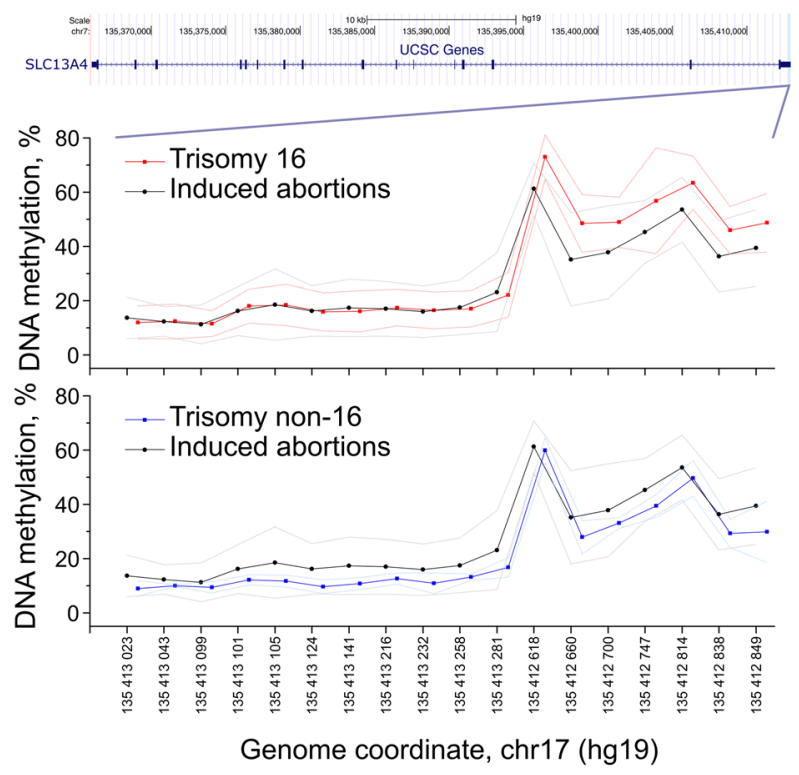

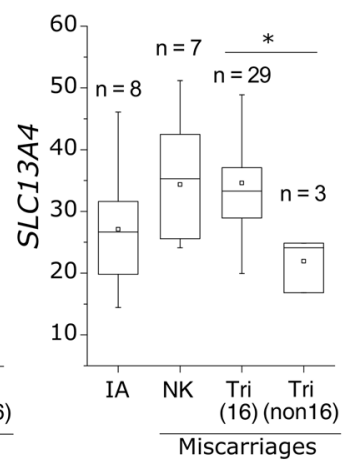

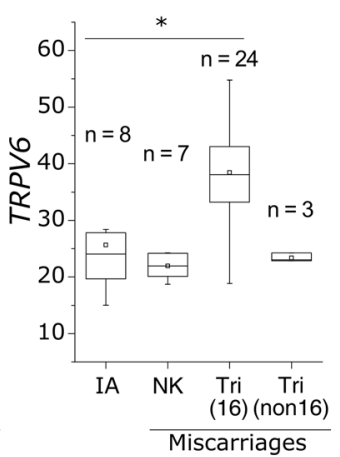

GATA3-AS1

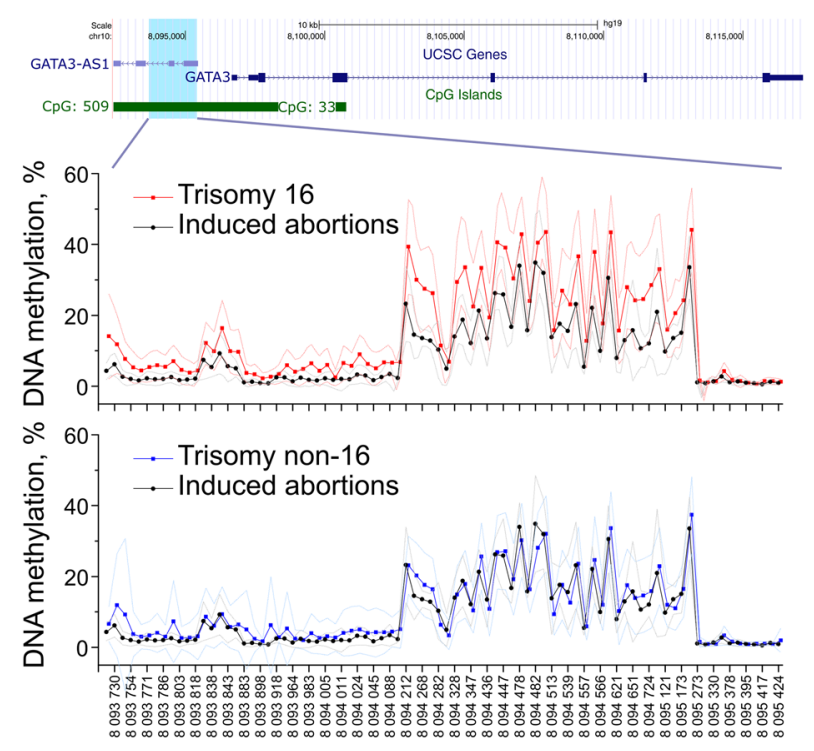

Genome coordinate, chr10 (hg19)

\section{TRVP6}
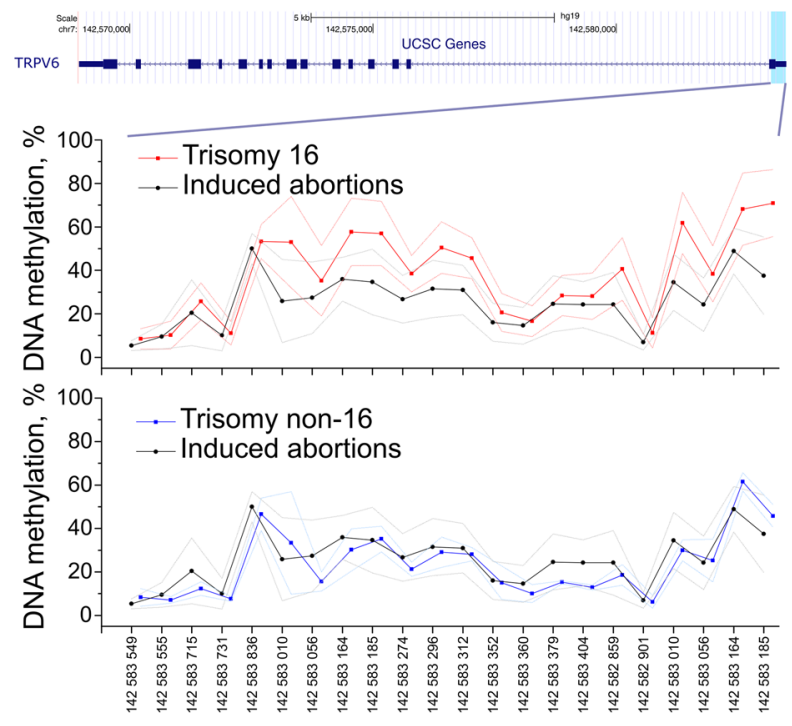

Genome coordinate, chr7 (hg19) 
4Figure 3. The CpG site methylation indices in $5 \mathrm{DMGs}$ in chorionic villi in the group of miscarriages with trisomy 16 compared to miscarriages with trisomy of other chromosomes, miscarriages with a normal karyotype, and induced abortions. (a) The average methylation indices for all analyzed CpG sites in the ANKRD53, GATA3-AS1, CALCB, SCL13A4, and TRPV6 genes in the groups of miscarriages with trisomy 16 (Tri (16)), miscarriages with trisomy of other chromosomes (Tri (non16)), miscarriages with a normal karyotype (NK), and induced abortions (IAs). The line in the center of the box marks the median. The boxes mark the 25th and 75th percentiles. The whiskers extend to the minimum and maximum values. The MannWhitney $\mathrm{U}$ test was used to compare groups. ${ }^{\star}-\mathrm{p}<0.05$. (b) A detailed profile of the methylation of CpG sites in the analyzed regions of the ANKRD53, GATA3-AS1, SCL13A4, and TRPV6 genes (indicated by a blue shaded area) in the groups of miscarriages with trisomy 16 and miscarriages with trisomy of other chromosomes compared to the induced abortion group. CpG islands are indicated by green bars. The dotted lines depict the standard deviation of the methylation profile. The figure was created using UCSC Genome Browser ${ }^{11}$.

lead to an increase in the frequency of anaphase lagging of various chromosomes and not only supernumerary chromosomes. The second hypermethylated gene, CDKN1A, encodes an inhibitor of the cyclin-dependent kinase $\mathrm{p} 21$. Increased expression of p21 serves as a cellular defense mechanism for suppressing further chromosomal instability and tumor development, and reduced expression following CDKN1A knockout leads to chromosomal instability ${ }^{30}$. Therefore, hypermethylation of this gene may also initiate the process of trisomy rescue.

The conclusions of our study have some limitations related to the tools used, the study design and the stages of development studied. The methylation index of $\mathrm{CpG}$ sites was analyzed only in chorionic villi, and the results obtained cannot be extrapolated to embryo tissues. Because only miscarriages at 6-10 weeks of development were included in our study, we could not distinguish between epigenetic disorders that originate in earlier stages of embryonic development, such as demethylation errors at the cleavage stage or when establishing a tissue-specific methylation pattern. The platform used for differential methylation analysis also imposes some limitations. The Illumina Infinium HumanMethylation27 BeadChip platform has a nonuniform genome coverage density, and the identified differentially methylated $\mathrm{CpG}$ sites are predominantly located in the promoter regions of genes. Therefore, the identification of the methylation profile in intergenic regions and gene bodies requires further studies using bisulfite whole genome sequencing. The identification of differentially methylated sites in genes does not always reflect changes in their expression and requires additional verification using functional approaches. The present study only analyzed changes in the expression of the GATA3 gene. Functional studies of the role of other identified differentially methylated genes in embryonic development will help elucidate the mechanisms of normal interactions between the embryo and the mother in the first trimester of pregnancy.

The present study confirmed that trisomy 16 was associated with large-scale methylation defects in chorionic villi and identified genes with aberrant methylation that may disrupt normal trophoblast differentiation and invasion and the normal mother-fetus interaction. We also identified genes that may initiate the process of mitotic instability and correction of trisomy 16.

\section{Material and methods}

Materials and karyotyping. We used samples of chorionic villi from 14 first-trimester miscarriages with trisomy 16 and 6 induced abortions, for which a genome-wide analysis of methylation using the Infinium HumanMethylation27 BeadChip (Illumina, USA) was previously performed (Supplementary Table S2) ${ }^{14}$. The methylation index of individual genes was analyzed in chorionic villi of 29 first-trimester miscarriages with trisomy 16 (Supplementary Table S2) using targeted bisulfite massive parallel sequencing. The DNA methylation index was also analyzed in 22 miscarriages with other aneuploidies, 7 miscarriages with normal karyotypes, and 8 induced abortions (Table 3 ).

Chorionic villi were separated from other extraembryonic and maternal tissues via morphology under the control of an inverted microscope. A suspension of cells was prepared from the chorionic villi samples, part of which was used to confirm the karyotype using FISH analysis, and DNA was extracted from the other part to determine the level of DNA methylation. Therefore, FISH analysis and DNA methylation analysis were performed from a common mixture of cells and not from different sections of the chorionic villi.

All of the women signed informed consent forms. The local Research Ethics Committee of the Research Institute of Medical Genetics granted their approval (22.04.2010/No 2). Before the start of the study, the samples were stored at $-80{ }^{\circ} \mathrm{C}$ without thawing. For all miscarriages, conventional cytogenetic analysis was performed on trophoblast cells or cultured extraembryonic fibroblasts, as described previously ${ }^{31}$. When the conventional cytogenetic analysis was noninformative, karyotypes were assessed using chromosomal comparative genome hybridization ( $\mathrm{cCGH}, \mathrm{n}=6)$ or array comparative genome hybridization $(\mathrm{aCGH}, \mathrm{n}=4)$ (Fig. 1) as described elsewhere ${ }^{15}$.

FISH. The karyotyping results in 29 miscarriages with trisomy 16 were validated using interphase FISH. The degree of mosaicism of trisomy 16 was assessed simultaneously with lower and upper thresholds of $10 \%$ and $90 \%$, respectively. Two-color subtelomeric (16p and 16q) DNA probes were used for analysis (Fig. 1). DNA probes were kindly provided by Prof. Mariano Rocchi at the University of Bari, Italy. FISH was performed as previously described ${ }^{32}$. Thirteen miscarriages had mosaicism of trisomy 16 (aneuploid clone-from 10 to $89 \%$ ), and 16 miscarriages had pure trisomy 16 (aneuploid clone $\geq 90 \%$ ).

Genome-wide DNA methylation analysis. Chorionic villous trophoblast cells were enriched via maceration of chorionic villi in $70 \%$ acetic acid using a modified protocol ${ }^{33}$. The isolated cells were washed in phos- 
a

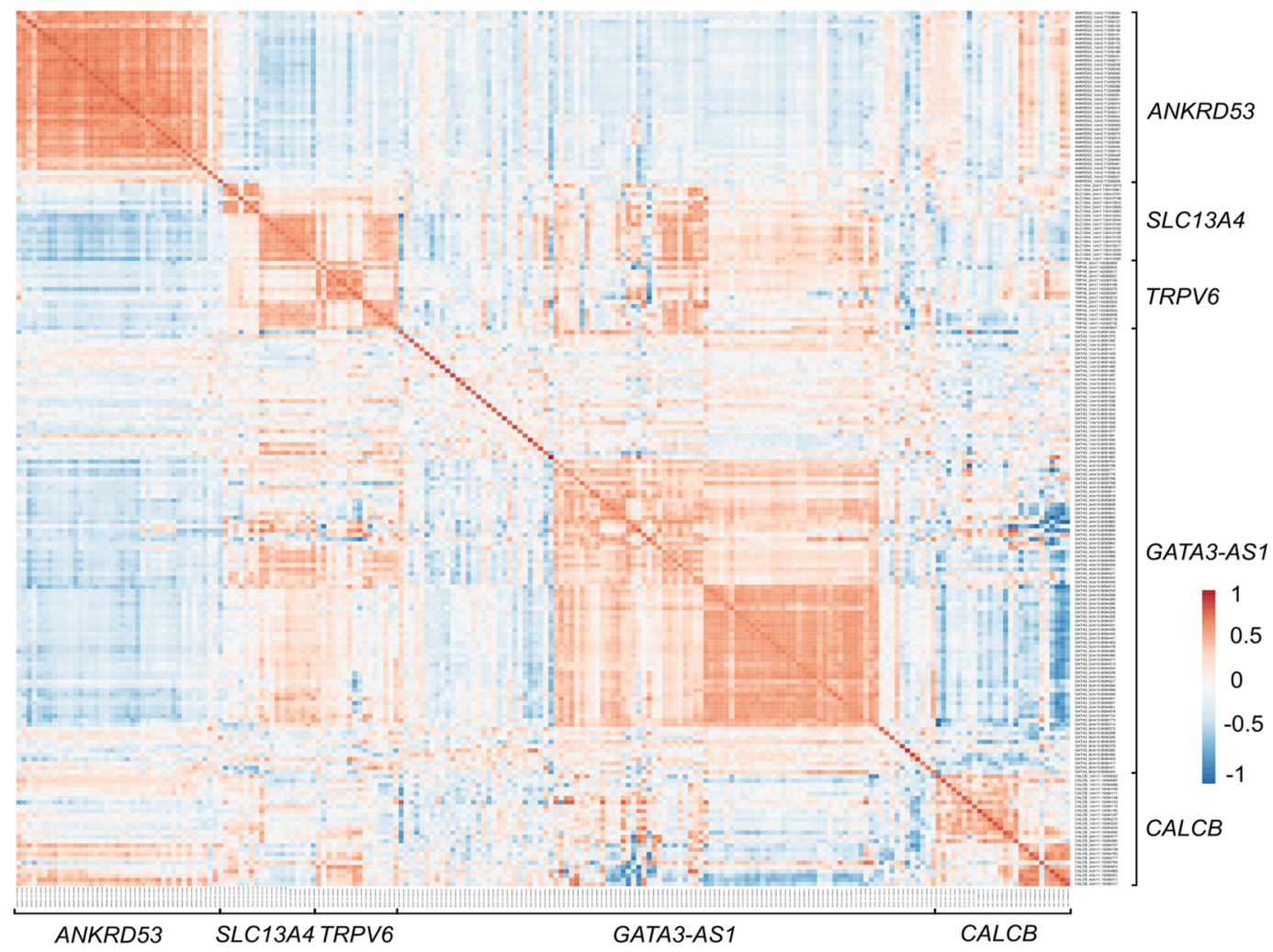

b

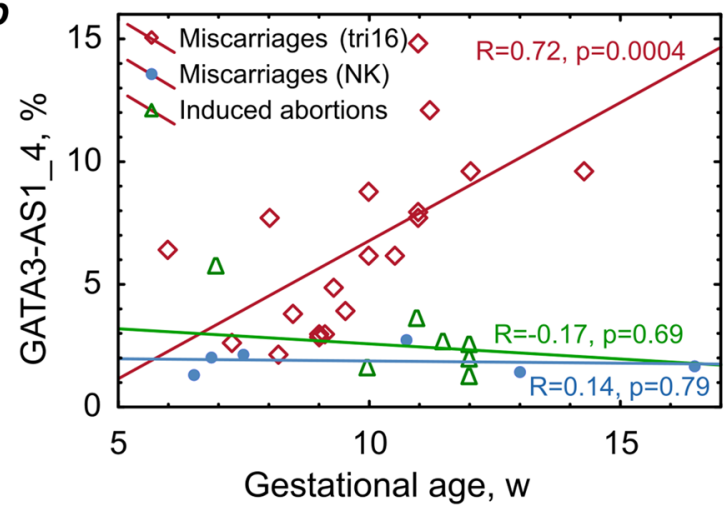

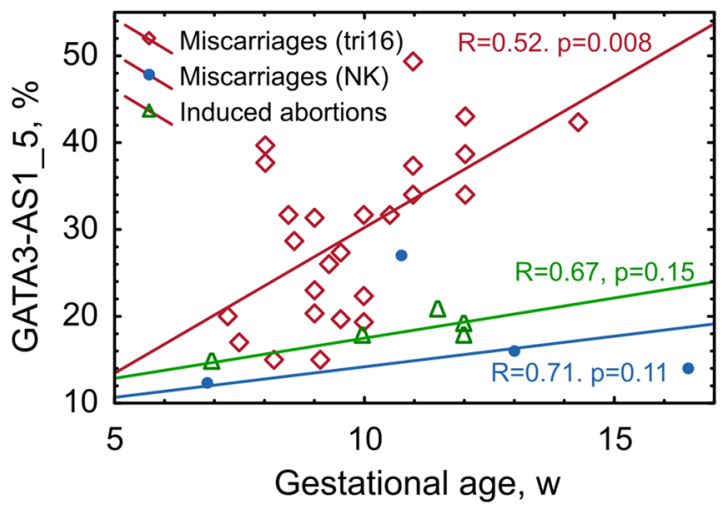

C

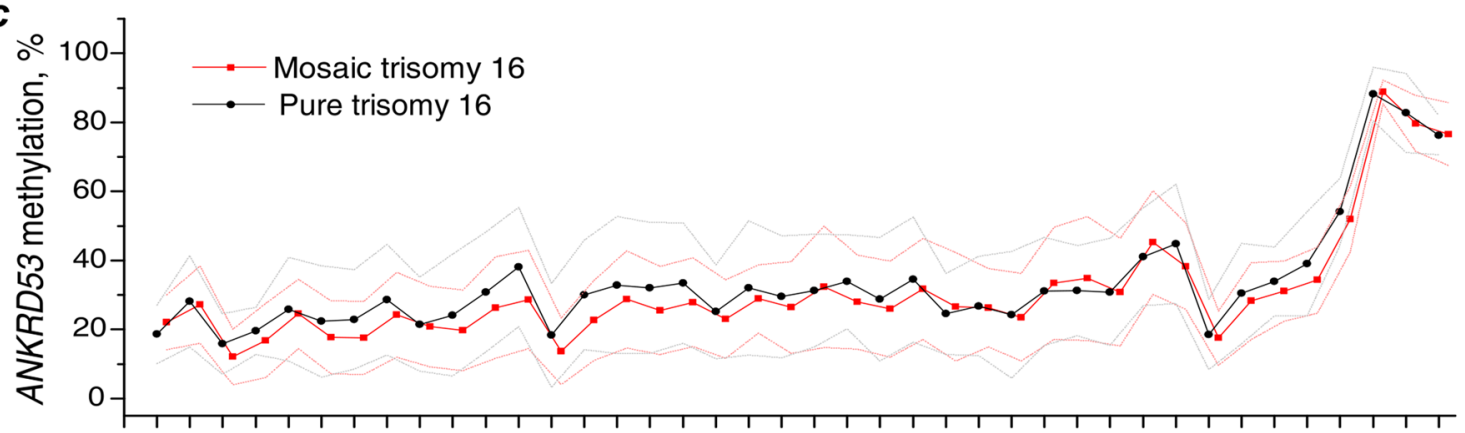

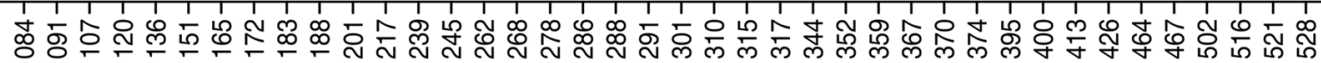

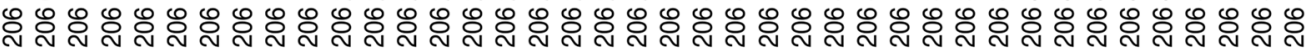

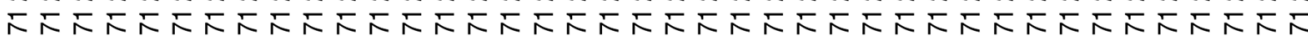

Genome coordinate, chr2 (hg19)

Figure 4. Correlation of the methylation indices of differentially methylated gene promoters with each other and their dependence on gestational age and chromosome mosaicism. (a) Correlation of the methylation indices of the ANKRD53, TRPV6, GATA3-AS1, SCL13A4, and CALCB genes with each other in chorionic villi of spontaneous abortions with trisomy 16. The heatmap was generated using the Spearman test and the ClustVis tool. (b) Correlation of the methylation indices of the GATA3-AS1_4-5 regions with the gestational age of miscarriages with trisomy 16, miscarriages with a normal karyotype, and induced abortions. (c) Comparison of the ANKRD53 gene methylation index in chorionic villi between groups of miscarriages with mosaic and pure trisomy 16. 
Trisomy 16

\begin{tabular}{ccccc}
\hline Group & Sample & \multicolumn{2}{c}{ Syncytiotrophoblast } & Cytotrophoblast \\
\hline \multirow{3}{*}{$\operatorname{tri16}$} & 1 & - & - \\
\cline { 2 - 4 } & 2 & + & ++ \\
\cline { 2 - 4 } & 3 & + & - \\
\cline { 2 - 4 } & 4 & + & +++ \\
\hline \multirow{2}{*}{ tri22 } & 1 & +++ & +++ \\
\cline { 2 - 4 } & 2 & +++ & ++ \\
\hline \multirow{3}{*}{ NK } & 1 & +++ & +++ \\
\cline { 2 - 4 } & 2 & + & +++ \\
\cline { 2 - 4 } & 3 & + & + \\
\cline { 2 - 4 } & 4 & + & ++ \\
\hline
\end{tabular}

Normal karyotype

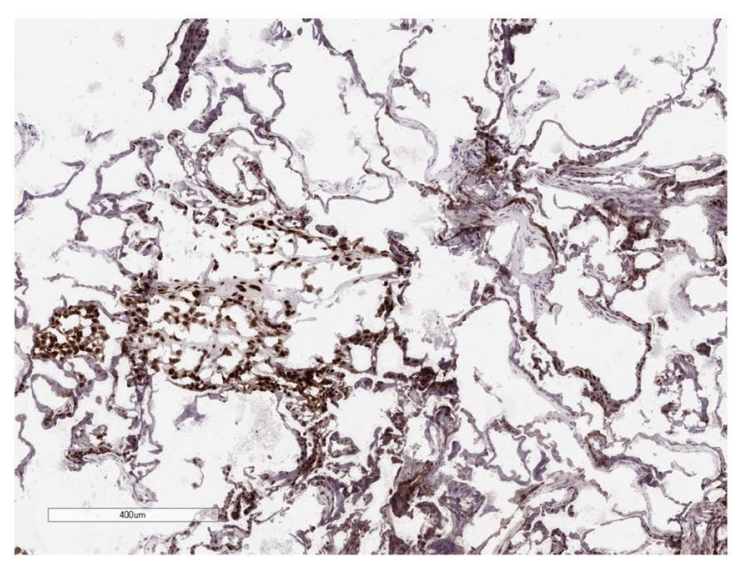

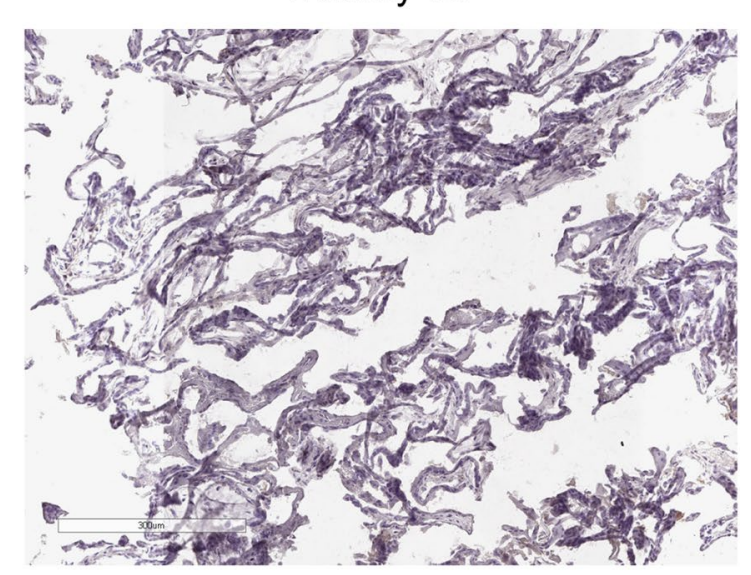

Trisomy 22

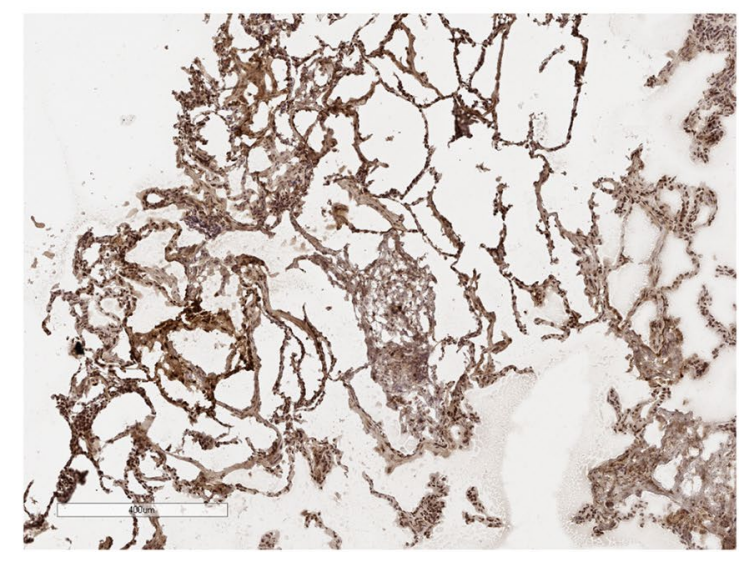

Figure 5. Examples of GATA3 protein staining in frozen sections of syncytiotrophoblast and cytotrophoblast cells of miscarriages with trisomy $16(n=4)$, trisomy $22(n=2)$, and a normal karyotype $(n=4)$.

\begin{tabular}{|l|l|l|l|l|l|}
\hline \multirow{2}{*}{ Group } & \multirow{2}{*}{} & Microarray analysis & \multicolumn{2}{l|}{ Targeted bisulfite massive parallel sequencing } \\
\cline { 3 - 7 } & Aneuploidy & Sample size & Gestational age, mean \pm SD, (min-max), w & Sample size & Gestational age, mean \pm SD, $(\mathbf{m i n}-\mathbf{m a x}), \mathbf{w}$ \\
\hline Miscarriages with trisomy 16 & Trisomy 16 & 12 & $8.4 \pm 2.0(6-12)$ & 29 & $9.4 \pm 1.8(6-12)$ \\
\hline Miscarriages with other aneuploidy & See Table S2 & - & - & 22 & $8.9 \pm 1.4(6.5-11.5)$ \\
\hline Miscarriages with a normal karyotype & - & - & - & 7 & $10.1 \pm 3.6(6.5-16.5)$ \\
\hline Induced abortions & - & 6 & $8.8 \pm 1.9(7-12)$ & 8 & $9.4 \pm 2.7(7-12)$ \\
\hline
\end{tabular}

Table 3. Characteristics of miscarriages with a normal karyotype, miscarriages with aneuploidy and induced abortions.

phate buffer. Genomic DNA was isolated from chorionic villi using the phenol-chloroform method. DNA methylation profiles were determined using the Infinium HumanMethylation27 BeadChip (Illumina, USA), which includes 27,578 CpG sites in 14,475 genes, according to the manufacturer's protocol. The data were analyzed using the software package GenomeStudio Methylation Module (Illumina), which translates the fluorescence intensity into a quantitative value of $\beta$ (methylation index) that corresponds to the ratio of the fluorescence signals of methylated alleles to the sum of the signals of methylated and unmethylated alleles of the studied locus. The absolute difference in the means of the $\beta$ values between compared groups was calculated for each $\mathrm{CpG}$ within a region and referred to as the delta beta $(\Delta \beta)$ value.

Analysis of DNA methylation microarray data was performed using the Bioconductor lumi ${ }^{34}$ and limma ${ }^{35}$ packages in the R statistical environment. Briefly, color bias correction and quantile normalization were used for data preprocessing. Differentially methylated CpG sites in chorionic villi between miscarriages with trisomy 16 and induced abortions were assessed using an empirical Bayes moderated t-test ${ }^{36}$. The Benjamini-Hochberg test was used to adjust the p values for the FDR. Enrichment analysis of the list of genes with differentially methylated CpG sites was performed using DAVID software ${ }^{37}$. Functional relationships between DMGs and genes involved in placental development were analyzed using the STRING tool ${ }^{38}$. 
Targeted bisulfite massive parallel sequencing. The DNA methylation index in the promoter regions of 5 genes (ANKRD53, TRPV6, GATA3-AS1, SCL13A4, and CALCB) was measured using bisulfite amplicon massive parallel sequencing. Sodium bisulfite conversion of genomic DNA was performed using an EZ DNA Methylation-Direct Kit (Zymo Research, USA). After bisulfite conversion of DNA, amplification of the products was performed using PCR with self-designed primers (Supplementary Table S3). The primers were designed using MethPrimer ${ }^{39}$, and their specificity was assessed using the BiSearch tool ${ }^{40}$. PCR was performed with BioMaster HS-Taq PCR-Color $(2 \times)$ PCR mix (BioLabMix, Russia). The following PCR conditions were used: primary denaturation, $15 \mathrm{~min}$ at $95^{\circ} \mathrm{C} ; 45$ cycles of $20 \mathrm{~s}$ at $95^{\circ} \mathrm{C}, 20 \mathrm{~s}$ at $50{ }^{\circ} \mathrm{C}$, and $20 \mathrm{~s}$ at $72{ }^{\circ} \mathrm{C}$; and terminal elongation, $5 \mathrm{~min}$ at $72^{\circ} \mathrm{C}$.

Amplified DNA was isolated using Sephadex G50 (Sigma, USA) and pooled for library generation. Adapters and indices were attached using the Nextera XT kit (Illumina) according to the manufacturer's protocol. Sequencing was performed on a MiSeq sequencer using the MiSeq Reagent Micro Kit v2 kit (Illumina, San Diego, CA, USA) according to the manufacturer's protocol.

For analysis of the targeted bisulfite sequencing results, the obtained reads were filtered in the Trimmomatic program with a Phred threshold of 30 . The filtered reads were aligned to in silico bisulfite-converted DNA in BWA-MEM. We counted the number of aligned nucleotides at each position and calculated the methylation index, the proportion of methylated cytosines, with SAMtools and $\mathrm{R}$ using the formula Methyl $=\mathrm{C} /(\mathrm{C}+\mathrm{T})$. The distribution of data was analyzed using the Kolmogorov-Smirnov test. The Mann-Whitney U test was used to compare groups. The Spearman test was used for correlation analyses.

Immunohistochemistry. Seven-micrometer-thick sections of frozen samples of chorionic villi of miscarriages with trisomy $16(n=4)$, trisomy $22(n=2)$, and normal karyotype $(n=4)$ stored in liquid nitrogen were mounted on poly-L-lysine slides (Thermo Fisher Scientific, Waltham, MA, USA). Briefly, the sections were fixed in paraformaldehyde (Sigma, St. Louis, MO, USA) for $5 \mathrm{~min}$ and washed twice in phosphate-buffered saline (PBS) for $5 \mathrm{~min}$. The sections were incubated for $5 \mathrm{~min}$ with a peroxidase blocking reagent (Agilent, Santa Clara, CA, USA) and washed twice in PBS for $5 \mathrm{~min}$. The sections were incubated for $15 \mathrm{~min}$ with a primary anti-GATA3 antibody (EP368, 1:100, Merck KGaA, Darmstadt, Germany) and washed twice in PBS for 5 min. The sections were incubated for 15 min with EnVision Flex/HRP (Agilent, Santa Clara, CA, USA), washed twice in PBS for $5 \mathrm{~min}$, and incubated for $5 \mathrm{~min}$ with a diaminobenzidine (DAB) solution (Agilent, Santa Clara, CA, USA). Nuclei were contrasted using hematoxylin. The sections were mounted according to a standard operating procedure. As a control for nonspecific binding, sections were incubated with an appropriate primary antibody or with only the secondary antibody. Slides were scanned using an Aperio AT2 (Leica, Wetzlar, Germany). Image analysis was performed using the ImageJ program using the IHC Profiler plugin ${ }^{41,42}$.

Ethics declarations. This study was performed in accordance with the principles of the Declaration of Helsinki. The local Research Ethics Committee of the Research Institute of Medical Genetics, Tomsk NRMC approved this study (22.04.2010/No 2).

\section{Data availability}

The datasets used and/or analyzed during the current study are available from the corresponding author on request.

Received: 30 April 2021; Accepted: 10 December 2021

Published online: 21 January 2022

\section{References}

1. Biesecker, L. G. \& Spinner, N. B. A genomic view of mosaicism and human disease. Nat. Rev. Genet. 14, 307-320. https://doi.org/ $10.1038 / \mathrm{nrg} 3424(2013)$.

2. Tolmacheva, E. N., Vasilyev, S. A. \& Lebedev, I. N. Aneuploidy and DNA methylation as mirrored features of early human embryo development. Genes (Basel) https://doi.org/10.3390/genes11091084 (2020).

3. Warburton, D., Byrne, J. M. \& Canki, N. Chromosome Anomalies and Prenatal Development: An Atlas. (Oxford University Press, 1991).

4. Benn, P. Trisomy 16 and trisomy 16 mosaicism: A review. Am. J. Med. Genet. 79, 121-133 (1998)

5. Sparks, T. N., Thao, K. \& Norton, M. E. Mosaic trisomy 16: What are the obstetric and long-term childhood outcomes?. Genet. Med. 19, 1164-1170. https://doi.org/10.1038/gim.2017.23 (2017).

6. Yong, P. J., Langlois, S., von Dadelszen, P. \& Robinson, W. The association between preeclampsia and placental trisomy 16 mosaicism. Prenat. Diagn. 26, 956-961. https://doi.org/10.1002/pd.1534 (2006).

7. Yong, P. J., Marion, S. A., Barrett, I. J., Kalousek, D. K. \& Robinson, W. P. Evidence for imprinting on chromosome 16: The effect of uniparental disomy on the outcome of mosaic trisomy 16 pregnancies. Am. J. Med. Genet. 112, 123-132. https://doi.org/10.1002/ ajmg.10702 (2002).

8. Schulze, K. V. et al. Novel parent-of-origin-specific differentially methylated loci on chromosome 16. Clin. Epigenet. 11, 60. https:// doi.org/10.1186/s13148-019-0655-8 (2019).

9. Blair, J. D., Langlois, S., McFadden, D. E. \& Robinson, W. P. Overlapping DNA methylation profile between placentas with trisomy 16 and early-onset preeclampsia. Placenta 35, 216-222. https://doi.org/10.1016/j.placenta.2014.01.001 (2014).

10. Hassold, T., Hall, H. \& Hunt, P. The origin of human aneuploidy: Where we have been, where we are going. Hum. Mol. Genet. 16(2), R203-R208. https://doi.org/10.1093/hmg/ddm243 (2007).

11. Raney, B. J. et al. Track data hubs enable visualization of user-defined genome-wide annotations on the UCSC Genome Browser. Bioinformatics 30, 1003-1005. https://doi.org/10.1093/bioinformatics/btt637 (2014)

12. Novakovic, B. et al. Evidence for widespread changes in promoter methylation profile in human placenta in response to increasing gestational age and environmental/stochastic factors. BMC Genomics 12, 529. https://doi.org/10.1186/1471-2164-12-529 (2011).

13. Gibbons, H. R. et al. Divergent lncRNA GATA3-AS1 regulates GATA3 transcription in T-helper 2 cells. Front. Immunol. 9, 2512. https://doi.org/10.3389/fimmu.2018.02512 (2018). 
14. Tolmacheva, E. N., Kashevarova, A. A., Skryabin, N. A. \& Lebedev, I. N. Epigenetic effects of trisomy 16 in human placenta. Mol. Biol. 47, 373-381. https://doi.org/10.1134/s0026893313030175 (2013).

15. Vasilyev, S. A. et al. LINE-1 retrotransposon methylation in chorionic villi of first trimester miscarriages with aneuploidy. J. Assist. Reprod. Genet. 38, 139-149. https://doi.org/10.1007/s10815-020-02003-1 (2021).

16. Du, J., Johnson, L. M., Jacobsen, S. E. \& Patel, D. J. DNA methylation pathways and their crosstalk with histone methylation. Nat. Rev. Mol. Cell Biol. 16, 519-532. https://doi.org/10.1038/nrm4043 (2015).

17. Pendina, A. A. et al. DNA methylation patterns of metaphase chromosomes in human preimplantation embryos. Cytogenet. Genome Res. 132, 1-7. https://doi.org/10.1159/000318673 (2011).

18. Lee, B. et al. Function and hormonal regulation of GATA3 in human first trimester placentation. Biol. Reprod. 95, 113. https://doi. org/10.1095/biolreprod.116.141861 (2016).

19. Paul, S., Home, P., Bhattacharya, B. \& Ray, S. GATA factors: Master regulators of gene expression in trophoblast progenitors. Placenta 60(Suppl 1), S61-S66. https://doi.org/10.1016/j.placenta.2017.05.005 (2017).

20. Jagtap, S. et al. All-trans retinoic acid and basic fibroblast growth factor synergistically direct pluripotent human embryonic stem cells to extraembryonic lineages. Stem Cell Research 10, 228-240. https://doi.org/10.1016/j.scr.2012.12.002 (2013).

21. Hoenderop, J. G. et al. Homo- and heterotetrameric architecture of the epithelial Ca2+ channels TRPV5 and TRPV6. EMBO J. 22, 776-785. https://doi.org/10.1093/emboj/cdg080 (2003).

22. Li, S. H. et al. TRPV5 and TRPV6 are expressed in placenta and bone tissues during pregnancy in mice. Biotechnic Histochem. 94, 244-251. https://doi.org/10.1080/10520295.2018.1548710 (2019).

23. Green, K. E., Thota, C., Hankins, G. D., Yallampalli, C. \& Dong, Y. L. Calcitonin gene-related peptide stimulates human villous trophoblast cell differentiation in vitro. Mol. Hum. Reprod. 12, 443-450. https://doi.org/10.1093/molehr/gal053 (2006).

24. Simmons, D. G. et al. Human placental sulfate transporter mRNA profiling from term pregnancies identifies abundant SLC13A4 in syncytiotrophoblasts and SLC26A2 in cytotrophoblasts. Placenta 34, 381-384. https://doi.org/10.1016/j.placenta.2013.01.017 (2013).

25. Rakoczy, J., Zhang, Z., Bowling, F. G., Dawson, P. A. \& Simmons, D. G. Loss of the sulfate transporter Slc13a4 in placenta causes severe fetal abnormalities and death in mice. Cell Res. 25, 1273-1276. https://doi.org/10.1038/cr.2015.100 (2015).

26. Torres, E. M. et al. Effects of aneuploidy on cellular physiology and cell division in haploid yeast. Science 317, $916-924$ (2007).

27. Williams, B. R. et al. Aneuploidy affects proliferation and spontaneous immortalization in mammalian cells. Science 322, 703-709. https://doi.org/10.1126/science.1160058 (2008).

28. Nicholson, J. M. et al. Chromosome mis-segregation and cytokinesis failure in trisomic human cells. Elife https://doi.org/10.7554/ eLife.05068 (2015).

29. Kim, S. \& Jang, C. Y. ANKRD53 interacts with DDA3 and regulates chromosome integrity during mitosis. Biochem. Biophys. Res. Commun. 470, 484-491. https://doi.org/10.1016/j.bbrc.2016.01.144 (2016).

30. Shen, K. C. et al. ATM and p21 cooperate to suppress aneuploidy and subsequent tumor development. Cancer Res. 65, 8747-8753. https://doi.org/10.1158/0008-5472.CAN-05-1471 (2005).

31. Lebedev, I. N., Ostroverkhova, N. V., Nikitina, T. V., Sukhanova, N. N. \& Nazarenko, S. A. Features of chromosomal abnormalities in spontaneous abortion cell culture failures detected by interphase FISH analysis. Eur. J. Hum. Genet. 12, 513-520. https://doi. org/10.1038/sj.ejhg.5201178 (2004).

32. Vasilyev, S. A., Timoshevsky, V. A. \& Lebedev, I. N. Cytogenetic mechanisms of aneuploidy in somatic cells of chemonuclear industry professionals with incorporated plutonium-239. Russ. J. Genet. 46, 1381-1385. https://doi.org/10.1134/s10227954101101 $41(2010)$.

33. Simoni, G. et al. Efficient direct chromosome analyses and enzyme determinations from chorionic villi samples in the first trimester of pregnancy. Hum. Genet. 63, 349-357. https://doi.org/10.1007/BF00274761 (1983).

34. Du, P., Kibbe, W. A. \& Lin, S. M. lumi: A pipeline for processing Illumina microarray. Bioinformatics 24, 1547-1548. https://doi. org/10.1093/bioinformatics/btn224 (2008).

35. Ritchie, M. E. et al. limma powers differential expression analyses for RNA-sequencing and microarray studies. Nucleic Acids Res. 43, e47. https://doi.org/10.1093/nar/gkv007 (2015).

36. Phipson, B., Lee, S., Majewski, I. J., Alexander, W. S. \& Smyth, G. K. Robust hyperparameter estimation protects against hypervariable genes and improves power to detect differential expression. Ann. Appl. Statist. 10, 946-963. https://doi.org/10.1214/16-AOAS9 20 (2016).

37. Jiao, X. et al. DAVID-WS: A stateful web service to facilitate gene/protein list analysis. Bioinformatics 28, 1805-1806. https://doi. org/10.1093/bioinformatics/bts251 (2012).

38. Szklarczyk, D. et al. STRING v11: Protein-protein association networks with increased coverage, supporting functional discovery in genome-wide experimental datasets. Nucleic Acids Res. 47, D607-D613. https://doi.org/10.1093/nar/gky1131 (2019).

39. Li, L. C. \& Dahiya, R. MethPrimer: Designing primers for methylation PCRs. Bioinformatics 18, 1427-1431 (2002).

40. Tusnady, G. E., Simon, I., Varadi, A. \& Aranyi, T. BiSearch: Primer-design and search tool for PCR on bisulfite-treated genomes. Nucleic Acids Res. 33, e9. https://doi.org/10.1093/nar/gni012 (2005).

41. Varghese, F., Bukhari, A. B., Malhotra, R. \& De, A. IHC Profiler: An open source plugin for the quantitative evaluation and automated scoring of immunohistochemistry images of human tissue samples. PLoS ONE 9, e96801. https://doi.org/10.1371/journal. pone.0096801 (2014).

42. Crowe, A. R. \& Yue, W. Semi-quantitative determination of protein expression using immunohistochemistry staining and analysis: An integrated protocol. Bio-Protoc. https://doi.org/10.21769/BioProtoc.3465 (2019).

\section{Acknowledgements}

The study was supported by the Russian Science Foundation (19-74-10026). Molecular cytogenetic and molecular genetic studies were performed in the "Medical Genomics" Core Facility of the Tomsk National Research Medical Center of the Russian Academy of Sciences using the resources of the biocollection "Biobank of the population of Northern Eurasia” of the Research Institute of Medical Genetics, Tomsk NRMC. We thank all of the families for their participation in the study. We are grateful to all of the clinicians involved in sample collection.

\section{Author contributions}

I.N., E.T., and S.V. conceptualized the study. E.T. and S.V. wrote the main manuscript text. E.T., S.V., T.N., E.L., E.S., D.Z., O.V., A.M., V.D., L.T., and A.K. performed the formal analyses and investigations. All authors reviewed the manuscript.

\section{Competing interests}

The authors declare no competing interests. 


\section{Additional information}

Supplementary Information The online version contains supplementary material available at https://doi.org/ 10.1038/s41598-021-04107-9.

Correspondence and requests for materials should be addressed to E.N.T.

Reprints and permissions information is available at www.nature.com/reprints.

Publisher's note Springer Nature remains neutral with regard to jurisdictional claims in published maps and institutional affiliations.

(c) (1) Open Access This article is licensed under a Creative Commons Attribution 4.0 International cc) License, which permits use, sharing, adaptation, distribution and reproduction in any medium or format, as long as you give appropriate credit to the original author(s) and the source, provide a link to the Creative Commons licence, and indicate if changes were made. The images or other third party material in this article are included in the article's Creative Commons licence, unless indicated otherwise in a credit line to the material. If material is not included in the article's Creative Commons licence and your intended use is not permitted by statutory regulation or exceeds the permitted use, you will need to obtain permission directly from the copyright holder. To view a copy of this licence, visit http://creativecommons.org/licenses/by/4.0/.

(C) The Author(s) 2022 